\title{
miR-124 inhibits cell growth through targeting IQGAP1 in colorectal cancer
}

\author{
JIANYU FAN ${ }^{1,2^{*}}$, WENJING ZHANG ${ }^{1,3^{*}}$, YANTING WU ${ }^{1,2}$, PING WAN $^{2,4}$, QIANG GUO ${ }^{2,4}$ and YU ZHANG ${ }^{2,4}$ \\ ${ }^{1}$ Faculty of Medicine, Kunming University of Science and Technology, Kunming, Yunnan 650000; \\ Departments of ${ }^{2}$ Gastroenterology and ${ }^{3}$ Medical Oncology, The First People's Hospital of Yunnan Province; \\ ${ }^{4}$ Yunnan Provincial Institute of Digestive Medicine, Kunming, Yunnan 650032, P.R. China
}

Received November 8, 2017; Accepted August 31, 2018

DOI: $10.3892 / \mathrm{mmr} .2018 .9518$

\begin{abstract}
MicroRNA (miRNA/miR)-124 is a miRNA, which exerts tumor suppressive effects but is frequently absent in tumors. Although it has been validated to target oncogenic genes such as signal transducer and activator of transcription 3, forkhead box Q1, and Slug, the mechanistic link between miR-124 and potential target genes that contribute to tumor progression, is yet to be investigated. IQ motif containing GTPase activating protein 1 (IQGAP1) is a scaffold protein that participates in protein-protein interactions and integrating diverse signaling pathways. Previous studies suggest that overexpression of IQGAP1 enhances activity of mitogen activated protein kinase 1 and $\beta$-catenin signaling cascades to facilitate tumor progression. The present study aimed to identify the regulative link between miR-124 and IQGAP1 in colorectal cancer (CRC). It was demonstrated that IQGAP1 was aberrantly overexpressed in CRC tissues and cell lines. Knockdown of IQGPA1 by introducing short hairpin-IQGAP1 lentivirus inhibited CRC cell growth and colony formation ability, and simultaneously suppressed phosphorylation of extracellular signal-regulated kinase (ERK) $1 / 2$ and $\beta$-catenin expression. Furthermore, it was demonstrated that miR-124 was silenced in CRC. Restoration of miR-124 in CRC cells impeded cell growth and colony formation ability. The direct binding of miR-124 to the 3'untranslated region of IQGAP1 mRNA was confirmed using a luciferase reporter gene assay. Importantly, downregulation of IQGAP1 expression was observed in miR-124-restoration cells with simultaneous reduction of
\end{abstract}

Correspondence to: Dr Yu Zhang or Professor Qiang Guo, Department of Gastroenterology, The First People's Hospital of Yunnan Province, 157 Jinbi Road, Kunming, Yunnan 650032, P.R. China

E-mail: yuzhang320@sina.com

E-mail: gqkj003@sina.com

*Contributed equally

Key words: microRNA-124, IQ motif containing GTPase activating protein 1, colorectal cancer, extracellular signal regulated kinase 1/2, $\beta$-catenin
phosphorylated-ERK $1 / 2$ and $\beta$-catenin. In conclusion, the present study describes a potential mechanism underlying the miR-124/IQGAP1 link in CRC progression. Silencing of miR-124 may depress IQGAP1 expression, leading to increased activity of ERK1/2 and $\beta$-catenin signaling.

\section{Introduction}

MicroRNAs (miRNA/miR) represent a cluster of small non-coding RNA molecules involved in controlling gene expression, translation and cellular biological behaviors. In recent years, growing evidence suggests that miRNAs serve a crucial role in tumor development by functioning as oncogenes or tumor suppressors, depending on their target genes and downstream signaling pathways (1). Of these, miR-124, first identified to be highly expressed in the brain, has been disclosed to be aberrantly downregulated or silenced in multiple tumors including liver, breast, cervical, nasopharyngeal and lung cancer (2-6), which arises at least partly from DNA methylation (7-9). Furthermore, miR-124 has also been demonstrated to suppress cell growth, invasion, epithelial-mesenchymal transition, and even metastasis through targeting a series of oncogenes, including rho-associated protein kinase, EZH2, signal transducer and activator of transcription 3 (STAT3), Slug and forkhead box protein Q1 (FOXQ1) $(3,5,10,11)$. Given that miR-124 regulates various malignant phenotypes in tumor development by repressing different target genes, it is helpful to understand the role of miR-124 regulatory networks in tumors in order to identify novel miR-124-targeting genes.

Colorectal cancer (CRC) represents one of the leading causes of cancer-associated mortality worldwide (12). The expression of miR-124 is negatively correlated with grade of CRC differentiation, but positively associated with poor prognosis, therefore represents an independent prognostic factor for CRC $(13,14)$. Previous studies have demonstrated the tumor-suppressive properties of miR-124 in the regulation of tumor growth and metastasis (15-17). In addition, miR-124 has been demonstrated to sensitize the response of tumor cells to radiotherapy in $\mathrm{CRC}$, through inhibiting paired mesoderm homeobox protein 1 (18). Notably, an integrating analysis demonstrated that miR-124 mediates crosstalk within the Toll-like receptor signaling pathway in Crohn's disease, ulcerative colitis and CRC (19), which indicates that the 
involvement of miR-124 in CRC progression begins at a very early stage of pathology.

IQ motif containing GTPase activating protein 1 (IQGAP1) is one of the largest known scaffold proteins, participating in protein-protein interactions and integrating diverse signaling pathways. Evidence implicates IQGAP1 as an essential regulator of the mitogen activated protein kinase 1 (MAPK) (20-22) and Wnt/ $\beta$-catenin signaling pathways (23-25) that serve crucial roles in the progression of multiple tumors. IQGAP1 is demonstrated to be overexpressed in CRC tissues compared with the normal counterparts (26) and tends to be expressed more at the invasive front than at the upper portions within the carcinoma tissues, which is associated with higher rates of distant metastasis $(26,27)$. Notably, in silico predictions indicate that miR-124 has a conserved binding site within the 3'untranslated region (UTR) of IQGAP1 mRNA. Therefore, it was hypothesized that overexpression of IQGAP1 in CRC is, at least partly, due to silencing of miR-124.

\section{Materials and methods}

Tissue samples and cell lines. A panel of 30 pairs of primary CRCs and their matched adjacent normal mucosa, were obtained from patients (16 males and 14 females; aged 31-84 years) who underwent surgical resections, between June 2016 to December 2016 at the First People's Hospital of Yunnan province (Kunming, China). Tissues were snap-frozen in liquid nitrogen immediately after resection and then stored at $-80^{\circ} \mathrm{C}$ for further use. The clinical features of all $30 \mathrm{CRC}$ patients, including clinical stage, tumor location and treatment prior to surgical resection are presented in Table I. All patients whose tissue samples were collected for the study signed the informed consent. This project was approved by the Ethics Committee of the First People's Hospital of Yunnan Province.

Human CRC cell lines, including HT29, SW480, SW620, DLD1, LoVo and HCT116, and 293T cells, were purchased from the Cell Bank of the Chinese Academy of Sciences (Shanghai, China). CRC cells were maintained routinely in RPMI-1640 medium (Invitrogen; Thermo Fisher Scientific, Inc., Waltham, MA, USA) supplemented with $10 \%$ fetal bovine serum (FBS; Gibco; Thermo Fisher Scientific, Inc.) and 1\% penicillin-streptomycin solution. The normal colonic epithelial cell line FHC was kindly donated by Dr. Liang Peng from Guangzhou Medical University (Guangzhou, China). FHC cells were maintained in Dulbecco's modified Eagle's medium (DMEM): F12 medium (Gibco; Thermo Fisher Scientific, Inc.) with supplements following ATCC protocol. 293T cells were maintained in DMEM medium containing high glucose supplemented with $10 \%$ FBS. All the cells mentioned were cultured in a $37^{\circ} \mathrm{C}$ humidified atmosphere containing $5 \% \mathrm{CO}_{2}$.

Bioinformatics analyses. The analysis of the microarray dataset (accession no. GSE20916) (28) was performed using bioinformatics tool R2 (http://r2.amc.nl) following the manufacturer's protocol. The 2 subgroups of normal colon tissues differed in collection method. One consisted of 24 samples is collected by surgery, the other of 10 samples is collected by colonoscopy. The colon tumor subgroup consisting of 30 samples were also from colonoscopy. Targetscan version 7.1 (http://targetscan.org), Pictar (http://pictar.mdc-berlin.de) and
miRecords (http://c1.accurascience.com/miRecords/) were used for prediction of miR-124 target genes.

Oligonucleotide and cell transfection. Hsa-miR-124 mimics and its scrambled controls were synthesized by Shanghai Genepharma Co., Ltd. (Shanghai, China). The sequences of the hsa-miR-124 mimics were as follows: Sense 5'-UAA GGCACGCGGUGAAUGCC-3' and antisense 5'-CAUUCA CCGCGUGCCUUAUU-3'. The sequences of the scramble controls were as follows: Sense 5'-UUCUCCGAACGUGUC ACGUTT-3' and antisense 5'-ACGUGACACGUUCGG AGAATT-3'. For transfection, cells were seeded into 6-well clusters at a density of $3 \times 10^{6}$ cells/well and transfected using Lipofectamine ${ }^{\circledR} 2000$ (Invitrogen; Thermo Fisher Scientific, Inc.) according to the manufacturer's protocol, with $30 \mathrm{nM}$ miR-124 mimics or scramble controls for $48 \mathrm{~h}$ at $37^{\circ} \mathrm{C}$ before further experimentation in assays or RNA/protein extraction.

Lentivirus packaging and stable cell line establishment. IQGAP1-knockdown and negative control lentivirus particles were packaged by co-transfecting $2.5 \mu \mathrm{g}$ IQGAP1-short hairpin (sh)RNA plasmids or shRNA scramble control plasmids (GeneCopoeia, Inc., Rockville, MD, USA) using psi-LVRU6GP vector as backbone (GeneCopoeia, Inc.) with lentiviral packaging plasmids into 293T cells, using Lenti-Pac ${ }^{\mathrm{TM}}$ HIV Expression Packaging Systems (GeneCopoeia, Inc.) according to the manufacturer's protocol. The target sequence for IQGAP1-shRNA \#1 and \#2 are as follows: 5'-GGTTAT CACCCTCATTCGTTC-3' and 5'-GGCTTATGAGTACCT TTGTCA-3'. For lentivirus infection, cells were incubated with viral supernatant in the presence of $8 \mu \mathrm{g} / \mathrm{ml}$ polybrene for $24 \mathrm{~h}$, followed by Puromycin (Invitrogen; Thermo Fisher Scientific, Inc.) selection until drug-resistant colonies became visible.

$R N A$ extraction and quantitative polymerase chain reaction $(q P C R)$. Total RNA was isolated from CRC tissues and cells using the TRIzol $^{\circledR}$ reagent (Invitrogen; Thermo Fisher Scientific, Inc.). Mature miR-124 expression in cells was determined using a Hairpin-it ${ }^{\mathrm{TM}}$ miRNAs qPCR kit (Shanghai Genepharma, Co., Ltd.). RNU6B was used as an endogenous control. IQGAP1 mRNA expression was determined by using SYBR green qPCR assay (Takara Bio, Inc., Otsu, Japan). The thermocycling conditions were as follows: Denaturation at $95^{\circ} \mathrm{C}$ for $3 \mathrm{~min}$, followed by 40 cycles of amplification at $95^{\circ} \mathrm{C}$ for $12 \mathrm{sec}$ and extension at $62^{\circ} \mathrm{C}$ for $40 \mathrm{sec}$. GAPDH was used as the endogenous control. The sequence of primers for IQGAP1 was as follows: Forward: 5'-GGGACCAACCAAAGTGTGTCAAC-3', reverse: 5'-CTG CTCATTATTGCCTGTCTTGGA-3'. Primer sequence for GAPDH: Forward: 5'-TGACTTCAACAGCGACACCCA-3', reverse: 5'-CACCCTGTTGCTGTAGCCAAA-3'. Data was analyzed using the $2^{-\Delta \Delta \mathrm{Cq}}$ method (29). This experiment was repeated 3 separate times.

Cell growth assay and cell colony formation assay. Cell growth was detected by using a Cell Counting Kit-8 (CCK-8; Dojindo Molecular Technologies, Inc., Kumamoto, Japan) according to the manufacturer's protocol. For colony formation assay, cells were trypsinized and plated on 6-well plates at 
Table I. Clinicopathological features of the 30 colorectal cancer patients analyzed in the study.

\section{Characteristics}

\begin{tabular}{|c|c|}
\hline \multicolumn{2}{|l|}{ Age } \\
\hline Mean (SD) & $6.1(14.1)$ \\
\hline Range & $31-84$ \\
\hline \multicolumn{2}{|l|}{ Sex } \\
\hline Male & 16 \\
\hline Female & 14 \\
\hline \multicolumn{2}{|l|}{ Stage } \\
\hline I & 3 \\
\hline II & 7 \\
\hline III & 16 \\
\hline IV & 4 \\
\hline \multicolumn{2}{|l|}{ Location } \\
\hline Rectum & 14 \\
\hline Colon & 16 \\
\hline \multicolumn{2}{|l|}{ Histology } \\
\hline Adenomatous carcinoma & 15 \\
\hline Mucinous carcinoma & 5 \\
\hline \multicolumn{2}{|l|}{ Differentiation } \\
\hline Well & 6 \\
\hline Moderate & 17 \\
\hline Poor & 7 \\
\hline \multicolumn{2}{|c|}{ Chemotherapy prior to resection } \\
\hline mFOLFOX6 & 2 \\
\hline Capecitabine & 2 \\
\hline No treatment & 26 \\
\hline
\end{tabular}

$\mathrm{SD}$, standard deviation.

a density of $3 \times 10^{2}$ cells/well and cultured at $37^{\circ} \mathrm{C}$ for 10 days. The colonies were stained with $0.1 \%$ crystal violet solution containing $80 \%$ methanol for $5 \mathrm{~min}$ at room temperature. The number of colonies defined as $>50$ cells/colony were counted at $\mathrm{x} 40$ magnification by using a light microscope. The assays were performed in triplicate of wells in 3 separate experiments.

Western blot analysis. Cultured cells were lysed in radioimmunoprecipitation assay buffer (Cell Signaling Technology, Inc., Danvers, MA, USA) with 1\% PMSF, for 15 min on ice Subsequently, cell lysates were centrifuged at $14,000 \mathrm{x} \mathrm{g}$ for $30 \mathrm{~min}$ and the supernatant was harvested. Protein concentration was determined using a bicinchoninic acid protein assay kit (Pierce; Thermo Fisher Scientific, Inc.). A total of 30 or $50 \mu \mathrm{g}$ protein/lane was loaded onto a $10 \%$ SDS-PAGE minigel and transferred onto a polyvinylidene difluoride membrane. Membranes were blocked using Tris-buffered saline containing 20\% Tween-20 and 5\% non-fat milk at room temperature for $2 \mathrm{~h}$. Following probing with 1:1,000 diluted anti-IQGAP1 (cat. no. 29016; Cell Signaling Technology, Inc.), anti-phosphorylated (p)-extracellular signal regulated kinase (ERK)1/2 (cat. no. 4370; Cell Signaling Technology, Inc.),
anti-ERK1/2 (cat. no. 4695; Cell Signaling Technology, Inc.) and anti- $\beta$-catenin (cat. no. 9562; Cell Signaling Technology, Inc.) respectively at $4^{\circ} \mathrm{C}$ overnight, the blots were subsequently incubated with anti-rabbit horseradish peroxidase-conjugated anti-immunoglobulin (Ig)G (cat. no. 7074; Cell Signaling Technology, Inc.) for $1 \mathrm{~h}$ at room temperature. Signals were visualized using ECL Substrates (Pierce; Thermo Fisher Scientific, Inc.). $\beta$-actin (1:3,000; cat. no. sc-47778; Santa Cruz Biotechnology, Inc., Dallas, TX, USA) was used as an endogenous protein for normalization. This experiment was repeated 3 separate times.

Luciferase reporter assay. A fragment of wild type 3'UTR of IQGAP1 containing the putative miR-124 binding site was amplified by PCR. The PCR product was sub-cloned into a psiCHECK-2 vector (Promega Corporation, Madison, WI, USA) immediately downstream of the luciferase gene sequence. A corresponding psiCHECK-2 construct containing the 3'UTR of IQGAP1 with a mutant seed sequence of miR-124 was also synthesized.

293 cells were plated in triplicate wells in 24-well clusters at a density of $1 \times 10^{5}$ cells/well, then co-transfected with $0.1 \mu \mathrm{g}$ psiCHECK-2 construct and $30 \mathrm{nM}$ miR-124 mimic or scramble control using Lipofectamine ${ }^{\circledR} 2000$ (Invitrogen; Thermo Fisher Scientific, Inc.). Following incubation for $48 \mathrm{~h}$ at $37^{\circ} \mathrm{C}$, luciferase activity was detected using a dual-luciferase reporter assay system (Promega Corporation) and normalized by Renilla activity. The experiment was repeated 3 separate times.

Statistical analysis. Statistical analyses were performed using the SPSS software, version 15.0 (SPSS, Inc., Chicago, IL, USA) and GraphPad Prism software version 6.01 (Graphpad Software, Inc., La Jolla, CA, USA). Comparisons of miR-124 and IQGAP1 expression between CRC tissues and paired adjacent colonic epithelial tissues were performed using a Wilcoxon's paired test. All data are presented as the mean \pm standard deviation. Comparisons among multiple groups were performed using one-way analysis of variance followed by Tukey's post hoc test. $\mathrm{P}<0.05$ was considered to indicate a statistically significant difference.

\section{Results}

IQGAPI expression is aberrantly upregulated in CRC. IQGAP1 has been reported to be overexpressed in multiple tumors, including CRC. Therefore gene expression profiles of human CRC cohorts were first analyzed to observe the status of IQGAP1 expression. As demonstrated in Fig. 1A, IQGAP1 was significantly upregulated in the cohort of CRC tissues compared with the other 2 subgroups of normal colon tissues collected by surgery and colonoscopy respectively $(\mathrm{P}<0.001)$. qPCR was performed to detect IQGAP1 mRNA level in clinical specimens of CRC. In the cohort of 30 cases of paired CRC tissues and adjacent colon epithelia, it was demonstrated that IQGAP1 was significantly upregulated in tumors compared with the matched normal epithelia $(\mathrm{P}=0.0062$; Fig. 1B). IQGAP1 protein levels in CRC cell lines: HCT116, HT29, SW480, SW620, LoVo and DLD1, are presented in Fig. 1C. Since HCT116 and SW480 cells expressed a high 
A

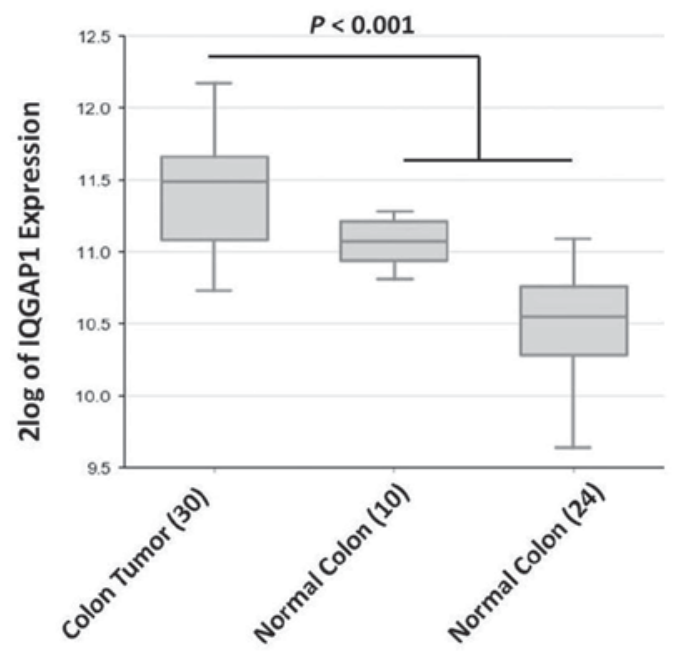

B

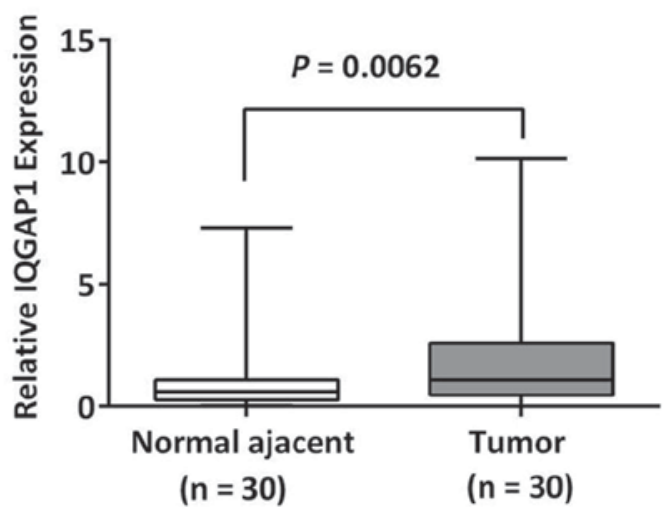

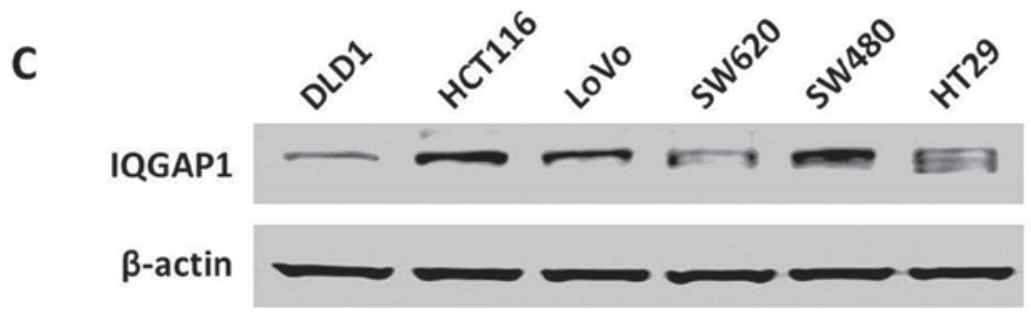

Figure 1. IQGAP1 is aberrantly overexpressed in CRC tissues and cell lines. (A) Bioinformatics analysis of the publicly available gene expression profile dataset GSE20916, revealed increased mRNA levels of IQGAP1 in primary CRC compared with the 2 subgroups of normal colon epithelia collected through surgery and colonoscopy respectively $(\mathrm{P}<0.001)$. (B) Upregulated IQGAP1 expression was observed in CRCs compared to the paired adjacent normal colon tissues from a panel of 30 CRCs. P=0.0062. (C) IQGAP1 protein level was measured in 6 CRC cell lines by western blotting. HCT116 and SW480 cells exhibited relatively higher expression of IQGAP1, therefore were selected for further loss-of-function assays. CRC, colorectal cancer; IQGAP1, IQ motif containing GTPase activating protein 1.

level of IQGAP1, they were selected for establishing stable IQGAP1-knockdown cells.

Knockdown of IQGAPI inhibits CRC cell growth and colony formation ability by suppressing phosphorylation of ERK and antagonizing $\beta$-catenin activity. Next, the role of IQGAP1 in CRC growth and the potential underlying mechanism was investigated. Stable IQGAP1 knockdown of CRC cells was generated by introducing IQGAP1 shRNA-packaged lentiviral particles into HCT116 and SW480 cells which express relatively higher levels of IQGAP1 compared with the other cell lines investigated in the present study. In the loss-of-function assays, downregulation of IQGAP1 significantly reduced cell growth $(\mathrm{P}<0.05)$ and colony formation ability (Fig. 2A-C). Immunoblotting demonstrated that in the IQGAP1-knockdown cells, phosphorylation of ERK1/2 was impaired as well as $\beta$-catenin activity, (Fig. 2D), demonstrating the augmenting effect of IQGAP1 on MAPK and $\beta$-catenin signaling.

miR-124 is aberrantly downregulated in CRC tissues. To demonstrate the expression pattern of miR-124 in CRC, qPCR was performed to detect the miR-124 level in the clinical specimens and cell lines. In the mentioned cohort of 30 cases of paired CRC tissues and adjacent colon epithelia, it was demonstrated that miR-124 was significantly downregulated in CRCs compared with the adjacent normal tissues $(\mathrm{P}=0.005$; Fig. 3A). The miR-124 expression level was further examined in CRC cell lines and miR-124 expression in all 6 CRC cell lines mentioned was significantly decreased compared with the normal colonic epithelial cell line FHC ( $\mathrm{P}<0.0001$; Fig. 3B). Notably, HCT116 and SW480 cells that expressed relatively high IQGAP1 expression levels (Fig. 1C), were demonstrated to express relatively low levels of miR-124 out of these CRC cell lines, therefore were chosen for restoration of miR-124 in further assays (Fig. 3B).

IQGAPl is a direct target gene of miR-124 in CRC. By utilizing bioinformatics algorithms (Targetscan/Pictar/miRecords), it was identified that IQGAP1 was a potential target gene of miR-124. The predicted binding site of miR-124 within the 3'UTR of IQGAP1 mRNA is presented in Fig. 4A. It was hypothesized that miR-124 may target IQGAP1 in CRC. To investigate this further, an miR-124 mimic was introduced into HCT116 and SW480 cells. With the restoration of miR-124 expression, IQGAP1 mRNA expression was demonstrated to be significantly decreased by qPCR $(\mathrm{P}<0.05)$. In addition, its protein level was demonstrated to be decreased by immunoblotting (Fig. 4B and C), while suppression of ERK1/2 phosphorylation and $\beta$-catenin was 
A

HCT-116

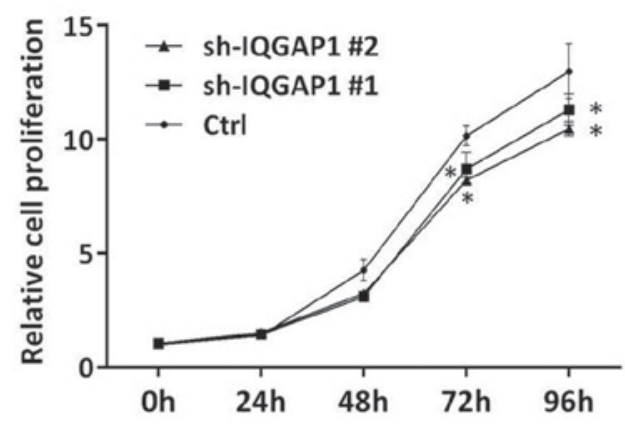

C

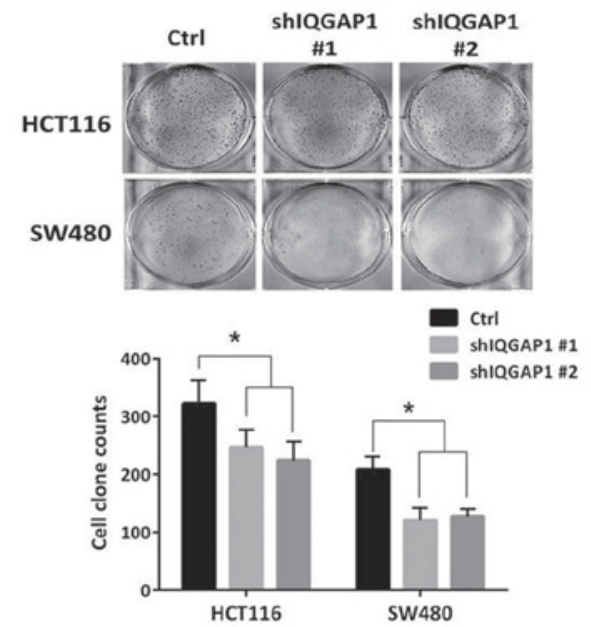

B

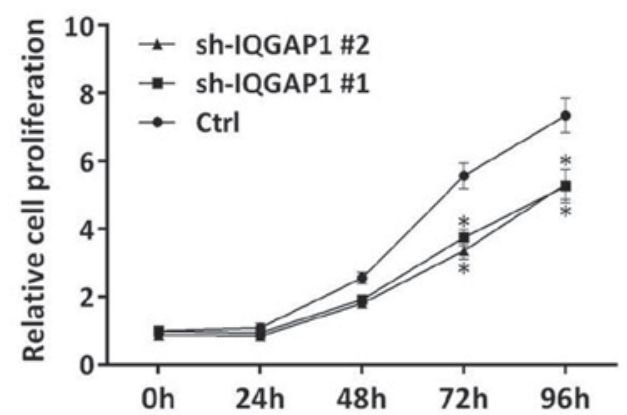

D

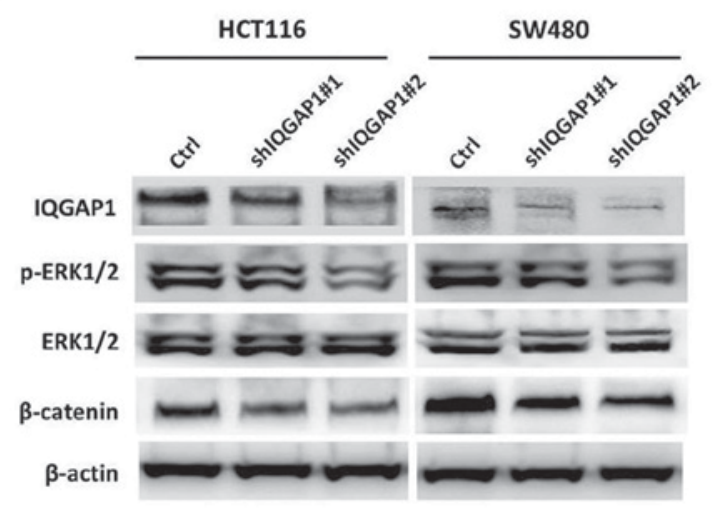

Figure 2. Knockdown of IQGAP1 inhibits CRC cell growth and colony formation ability. Knockdown of IQGAP1 in (A) HCT116 and (B) SW480 cells by introduction of IQGAP1-shRNA inhibited cell growth. * $\mathrm{P}<0.05$ vs. Ctrl. (C) Knockdown of IQGAP1 simultaneously inhibited colony formation ability in HCT116 and SW480 cells. ${ }^{*}$ P $<0.05$. (D) Knockdown of IQGAP1 in HCT116 and SW480 cells induced decreased phosphorylation of ERK1/2 and $\beta$-catenin expression demonstrated via western blotting. CRC, colorectal cancer; IQGAP1, IQ Motif Containing GTPase Activating Protein 1; sh, short hairpin; p, phosphorylated; ERK, extracellular signal regulated kinase; Ctrl, control.

A

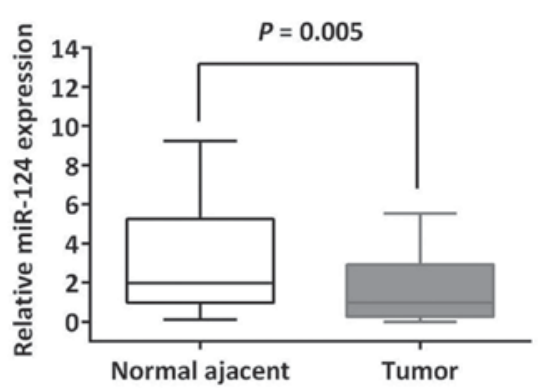

B

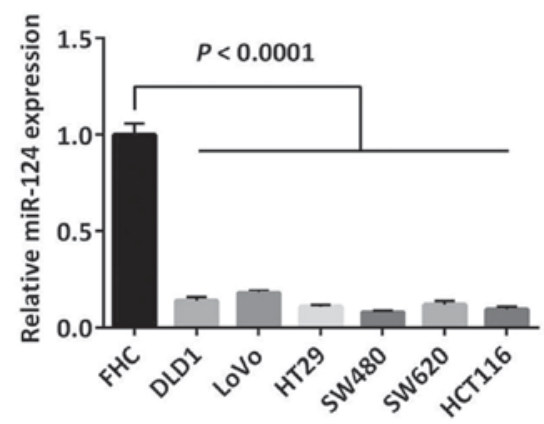

Figure 3. miR-124 is aberrantly downregulated in CRC tissues. (A) Expression of miR-124 is aberrantly downregulated in collected primary CRCs compared to the paired adjacent normal colon epithelia from the mentioned panel of $30 \mathrm{CRC}$ patients $(\mathrm{P}=0.005)$. (B) miR-124 expression is significantly decreased in 6 CRC cell lines compared with normal colonic epithelial cell line FHC (P<0.0001). HCT116 and SW480 cells demonstrate a low level of miR-124 out of these cell lines and were chosen for transfection of miR-124 mimics and further assays. miR, microRNA; CRC, colorectal cancer.

also simultaneously observed (Fig. 4C). To further investigate if the predicted binding site of miR-124 to 3'UTR of IQGAP1 mRNA is responsible for the downregulation of IQGAP1, the 3'UTR of IQGAP1 was cloned into a luciferase reporter vector wild-type (wt)-IQGAP1 and a corresponding mutant version (mut-IQGAP1) was also constructed. wt-IQGAP1 and miR-124 mimic or scrambled control were co-transfected into $293 \mathrm{~T}$ and HCT116 cells to perform a luciferase reporter assay. In $293 \mathrm{~T}$ and HCT116 cells, the luciferase activity of miR-124 transfected cells was significantly reduced compared to the scrambled control cells $(\mathrm{P}<0.05$; Fig. 4D and E). Furthermore, miR-124-mediated repression of luciferase activity was abolished by the mutant putative binding site (Fig. 4D and E). 
A

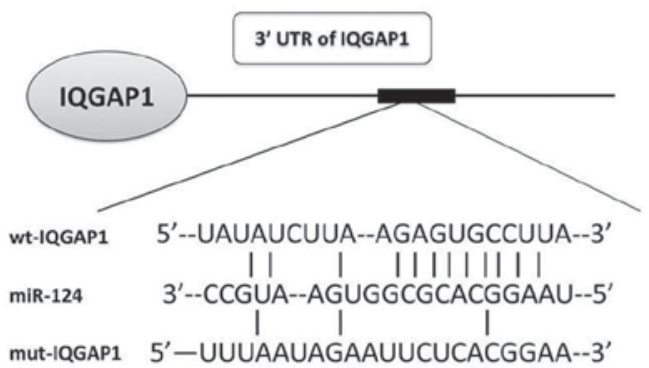

B

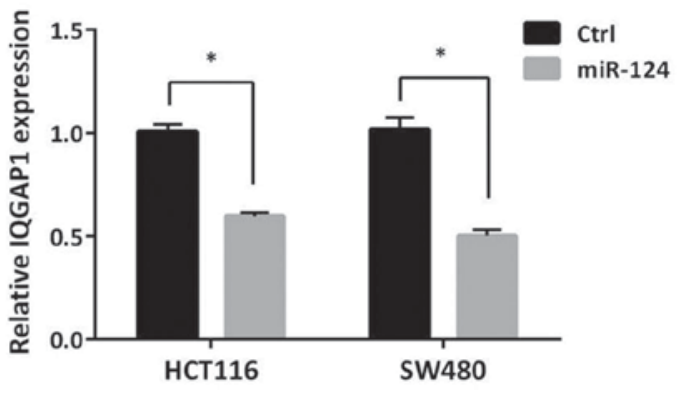

C

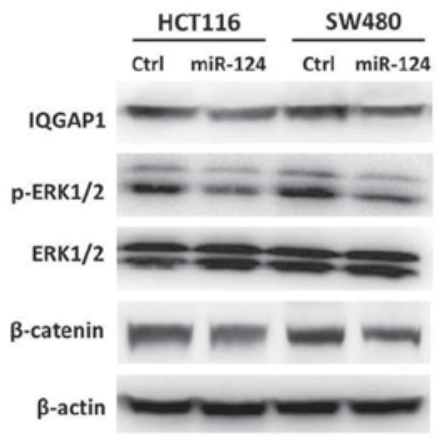

D

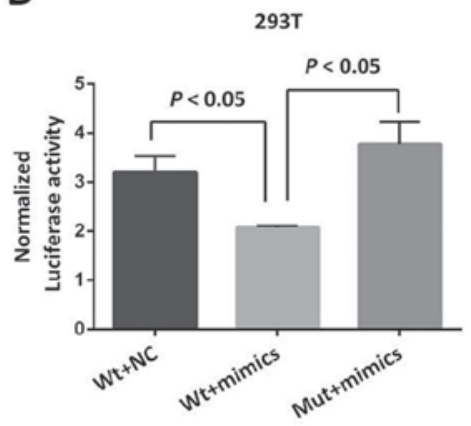

E

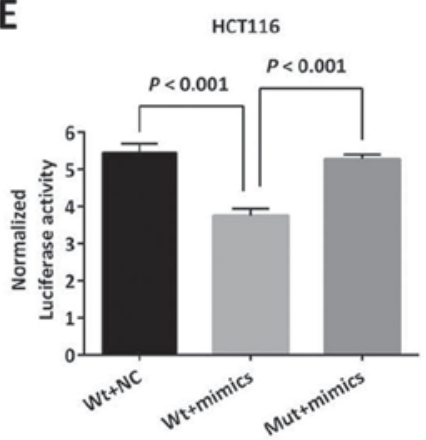

Figure 4. IQGAP1 is a direct target gene of miR-124 in CRC. (A) The predicted miR-124 binding site within IQGAP1 3'UTR and its mutated version are as presented. (B) Restoration of miR-124 in HCT116 and SW480 cells induced decreased expression of IQGAP1 by quantitative polymerase chain reaction. ${ }^{*} \mathrm{P}<0.05$ vs. the Ctrl. (C) Restoration of miR-124 in HCT116 and SW480 cells induced decreased phosphorylation of ERK1/2 and $\beta$-catenin expression. The repression of luciferase activity by wt-IQGAP1 3'UTR was dependent on miR-124, while mutated 3'UTR of IQGAP1 abrogated miR-124-mediated repression of luciferase activity in (D) $293 \mathrm{~T}$ and (E) HCT116 cells. miR, microRNA; CRC, colorectal cancer; IQGAP1, IQ motif containing GTPase activating protein 1; Ctrl, control; UTR, untranslated region; p, phosphorylated; ERK, extracellular signal regulated kinase; wt, wild-type; Mut, mutant; NC, negative control.

Restoration of miR-124 expression suppresses cell growth and colony formation ability of $C R C$. To investigate the effects of miR-124 on cell growth of CRC, the cell growth ability of miR-124-transfected HCT116 and SW480 cells was measured. First of all, significant restoration of miR-124 expression was performed by mimic transfection in the two cell lines, HCT116 and SW480 ( $\mathrm{P}<0.05$; Fig. 5A). CCK-8 assays demonstrated that restoration of miR-124 expression significantly repressed cell growth ability in HCT116 and SW480 cells after $72 \mathrm{~h}(\mathrm{P}<0.05$; Fig. 5B and C). Furthermore, significantly decreased colony formation activity was also observed in the two aforementioned cell lines $(\mathrm{P}<0.05$; Fig. 5D). These results suggested that miR-124 serves a suppressive role in tumor growth in CRC and its absence may confer malignant potentials to CRC cells.

\section{Discussion}

IQGAP1, the best characterized member of the IQGAP family, functions as a scaffold protein in the cytoplasm to curb, compartmentalize and coordinate multiple signaling pathways in a variety of cell types, and participates in cell-cell interaction, cell adherence, and movement via actin/tubulin-based cytoskeletal reorganization (30). In recent years, studies demonstrated that IQGAP1 is frequently aberrantly overexpressed in multiple tumors and integrates and mediates several pro-oncogenic signaling pathways to promote tumorigenesis and metastasis (31-34). IQGAP1 may bind directly to B-Raf, dual specificity mitogen-activated protein kinase kinase 1 and ERK, facilitating epidermal growth factor-induced activation of the MAPK cascade. IQGAP1 binding to $\beta$-catenin enhances $\beta$-catenin nuclear translocation, initiating transcription of cyclin D1 (35). Notably, a previous study by Jameson et al (36) verified that blockage of IQGAP1-ERK1/2 interactions by introducing a specific IQGAP1 WW domain peptide, inhibits RAS- and RAF-driven tumorigenesis and bypasses acquired resistance to the BRAF inhibitor, which validates IQGAP1 inhibition as a promising therapeutic strategy for tumors.

Dysregulation of miRNAs have been demonstrated to account for malignant phenotypes in tumors. Growing evidence proves that miR-124 is a miRNA, which exerts tumor suppressive effects but is frequently absent in tumors (2-6). Despite this miR-124 has been validated to target oncogenic genes including STAT3, FOXQ1 and Slug $(5,10,11)$ and the present study aimed to identify the mechanistic link of miR-124 to other potential target genes contributing to CRC development. Since IQGAP1 is predicted as a potential target gene of miR-124 by in silico prediction, it was hypothesized that there is a regulative link between miR-124 and IQGAP1. In the present study, IQGAP1 was demonstrated to be overexpressed in CRC tissue. Knockdown of IQGAP1 in CRC cells could impede cell growth and colony formation ability, which is considered to arise from repression of phosphorylated (p)-ERK1/2 and $\beta$-catenin expression. Similarly, restoration of miR-124 induced the inhibition of cell growth and colony formation ability, and repressed p-ERK1/2 and $\beta$-catenin. Given that miR-124 bound to the 3'UTR of IQGAP1 mRNA and led to a decrease of IQGAP1 
A

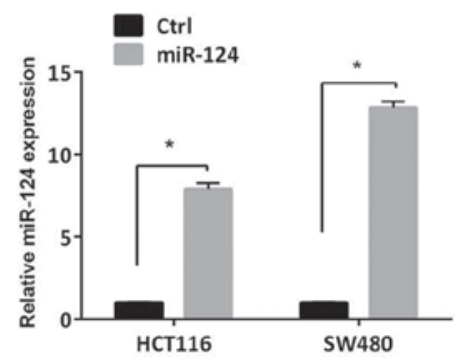

B

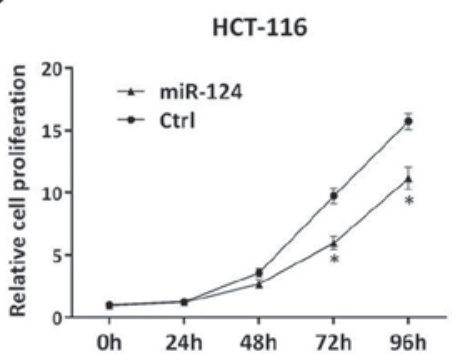

C

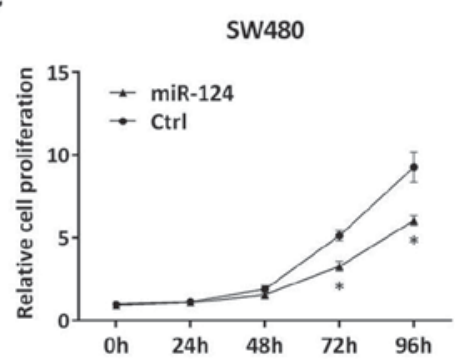

D
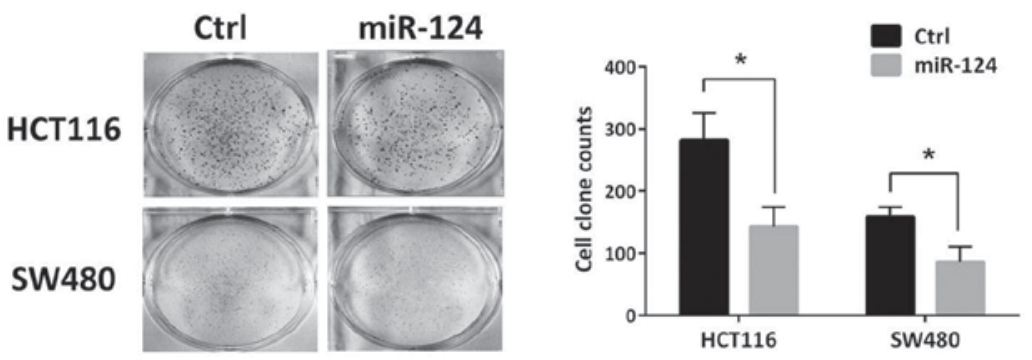

Figure 5. Restoration of miR-124 suppresses cell growth and colony formation ability of CRC. (A) miR-124 expression was significantly upregulated via mimic transfection in HCT116 and SW480 cells. "P<0.0001 vs. Ctrl. Restoration of miR-124 in (B) HCT116 and (C) SW480 cells by transfection of miR-124 mimic significantly inhibited cell growth. "P<0.05 vs. Ctrl (D) Restoration of miR-124 in HCT116 and SW480 cells simultaneously inhibits colony formation ability. ${ }^{*} \mathrm{P}<0.05$ vs. Ctrl. CRC, colorectal cancer; miR, microRNA; Ctrl, control.

expression, it was demonstrated that the absence of miR-124 was responsible, at least in part, for the aberrant upregulation of IQGAP1 and subsequent enhanced activation of MAPK and $\beta$-catenin signaling in CRC.

From the perspective of tumor treatment, it is urgent to investigate promising therapeutic targets and invent efficient approaches for tumor targeting. For example, the results of the present study indicated that IQGAP1 is a potential target for CRC treatment and further highlighted that miR-124 is in theory a potential way to antagonize IQGAP1 similar to the WW domain peptide of IQGAP1 mentioned. Recently, nanoparticles have emerged as a popular concept for targeted delivery of therapeutic agents to tumors (37-39). A type of combined polymeric nanoparticle (PNP) entrapping phosphatidyl inositol 3 kinase inhibitors or irinotecan suppresses or even eliminates lung metastasis of CRC in a BALB/C mice model, but negligible accumulation of PNPs is detected in the organs including the spleen, liver and kidneys indicating good tissue selectivity of PNPs and organ safety (40). Notably, delivery of nanoparticles conjugated with miR-20a or anti-miR-155 relieves aberrant expression of their protein targets and eventually reduces tumor growth and liver metastasis in murine colon cancer models $(41,42)$. In addition, considering miR-124 suppresses tumor progression through targeting a list of tumor-promoting proteins, miR-124-entrapped nanoparticles are anticipated to be a novel therapeutic option for treating tumors with absence of miR-124.

In conclusion, the present study provided evidence that accounted for aberrant upregulation of IQGAP1 in CRC and newly described a potential mechanism underlying dysregulated miR-124 indulging CRC progression. Therefore, it would be worthwhile to estimate the implication of miR-124-targeted therapy for clinical management of CRC.

\section{Acknowledgements}

The authors would like to thank Prof Liang Peng from Guangzhou Medical University (Guangzhou, China) for his technical assistance.

\section{Funding}

The present study was supported by grants from the National Natural Science Foundation of China (grant no. 81502128) and Joint Foundation of Kunming Medical University and Yunnan Provincial Science and Technology Department (grant no. 2014FB085).

\section{Availability of data and materials}

The dataset GSE20916 analyzed during the present study is publically available in GEO Datasets resource of NCBI [https://www.ncbi.nlm.nih.gov/gds/?term=GSE20916].

\section{Authors' contributions}

QG and YZ designed the study. JF, WZ and YW performed the experiments. PW analyzed the data and performed statistical analysis. YZ wrote the paper. All authors read and approved the manuscript.

\section{Ethics approval and consent to participate}

All patients whose tissue samples were collected for the study signed the informed consent. This project was approved by the Ethics Committee of the First People's Hospital of Yunnan Province. 


\section{Patient consent for publication}

All patients whose tissue samples were collected for the study signed an informed consent.

\section{Competing interests}

The authors declare that they have no competing interests.

\section{References}

1. Esquela-Kerschera A and Slack FJ: Oncomirs-microRNAs with a role in cancer. Nat Rev Cancer 6: 259-269, 2006.

2. Furuta M, Kozaki KI, Tanaka S, Arii S, Imoto I and Inazawa J: miR-124 and miR-203 are epigenetically silenced tumor suppressive microRNAs in hepatocellular carcinoma. Carcinogenesis 31: 766-776, 2010

3. Liang YJ, Wang QY, Zhou CX, Yin QQ, He M, Yu XT, Cao DX, Chen GQ, He JR and Zhao Q: MiR-124 targets Slug to regulate epithelial-mesenchymal transition and metastasis of breast cancer. Carcinogenesis 34: 713-722, 2013.

4. Wan HY, Li QQ, Zhang Y, Tian W, Li YN, Liu M, Li X and Tang H: MiR-124 represses vasculogenic mimicry and cell motility by targeting amotL1 in cervical cancer cells. Cancer Lett 355: 148-158, 2014

5. Peng XH, Huang HR, Lu J, Liu X, Zhao FP, Zhang B, Lin SX, Wang L, Chen HH, Xu X, et al: MiR-124 suppresses tumor growth and metastasis by targeting Foxq1 in nasopharyngeal carcinoma. Mol Cancer 13: 186, 2014

6. Zhang Y, Li H, Han J and Zhang Y: Down-regulation of microRNA-124 is correlated with tumor metastasis and poor prognosis in patients with lung cancer. Int J Clin Exp Pathol 8: $1967-1972,2015$

7. Murray-Stewart T, Sierra JC, Piazuelo MB, Mera RM, Chaturvedi R, Bravo LE, Correa P, Schneider BG, Wilson KT and Casero RA: Epigenetic silencing of miR-124 prevents spermine oxidase regulation: Implications for Helicobacter pylori-induced gastric cancer. Oncogene 35: 5480-5488, 2016.

8. Pronina IV, Loginov VI, Burdennyy AM, Fridman MV, Senchenko VN, Kazubskaya TP, Kushlinskii NE, Dmitriev AA and Braga EA: DNA methylation contributes to deregulation of 12 cancer-associated microRNAs and breast cancer progression. Gene 604: 1-8, 2017

9. Wang H, Zhang TT, Jin S, Liu H, Zhang X, Ruan CG, Wu DP, Han Y and Wang XQ: Pyrosequencing quantified methylation level of miR-124 predicts shorter survival for patients with myelodysplastic syndrome. Clin Epigenetics 9: 91, 2017.

10. Zheng F, Liao YJ, Cai MY, Liu YH, Liu TH, Chen SP, Bian XW, Guan XY, Lin MC, Zeng YX, et al: The putative tumour suppressor microRNA-124 modulates hepatocellular carcinoma cell aggressiveness by repressing ROCK 2 and EZH2. Gut 61 : 278-289, 2012

11. Cheng Y, Li Y, Nian Y, Liu D, Dai F and Zhang J: STAT3 is involved in miR-124-mediated suppressive effects on esophageal cancer cells. BMC Cancer 15: 306, 2015.

12. Jemal A, Siegel R, Xu J and Ward E: Cancer statistics, 2010. CA Cancer J Clin 60: 277-300, 2010.

13. Wang MJ, Li Y, Wang R, Wang C, Yu YY, Yang L, Zhang Y, Zhou B,Zhou ZG and Sun XF: Downregulation of microRNA-124 is an independent prognostic factor in patients with colorectal cancer. Int J Colorectal Dis 28: 183-189, 2013.

14. Jinushi T, Shibayama Y, Kinoshita I, Oizumi S, Jinushi M, Aota T, Takahashi T, Horita S, Dosaka-Akita H and Iseki K: Low expression levels of microRNA-124-5p correlated with poor prognosis in colorectal cancer via targeting of SMC4. Cancer Med 3: 1544-1552, 2014.

15. Taniguchi K, Sugito N, Kumazaki M, Shinohara H, Yamada N, Nakagawa Y, Ito Y, Otsuki Y, Uno B, Uchiyama K and Akao Y: MicroRNA-124 inhibits cancer cell growth through PTB1/PKM1/PKM2 feedback cascade incolorectal cancer. Cancer Lett 363: 17-27, 2015.

16. Chen Z, Liu S, Tian L, Wu M, Ai F, Tang W, Zhao L, Ding J, Zhang L and Tang A: miR-124 and miR-506 inhibit colorectal cancer progression by targeting DNMT3B and DNMT1. Oncotarget 6: 38139-38150, 2015.
17. Zhou L, Xu Z, Ren X, Chen K and Xin S: MicroRNA-124 (MiR-124) inhibits cell proliferation, metastasis and invasion in colorectal cancerby downregulating rho-associated protein kinase 1(ROCK1). Cell Physiol Biochem 38: 1785-1795, 2016

18. Zhang Y, Zheng L, Huang J, Gao F, Lin X, He L, Li D, Li Z, Ding Y and Chen L: MiR-124 Radiosensitizes human colorectal cancer cells by targeting PRRX1. PLoS One 9: e93917, 2014.

19. Bai J, Li Y, Shao T, Zhao Z, Wang Y, Wu A, Chen H, Li S, Jiang C, $\mathrm{Xu} \mathrm{J}$ and $\mathrm{Li} \mathrm{X}$ : Integrating analysis reveals microRNA-mediated pathway crosstalk among Crohn's disease, ulcerative colitis and colorectal cancer. Mol Biosyst 10: 2317-2328, 2014.

20. Roy M, Li Z and Sacks DB: IQGAP1 is a scaffold for mitogen-activated protein kinase signalling. Mol Cell Biol 25: 7940-7952, 2005

21. Roy M, Li Z and Sacks DB: IQGAP1 binds ERK2 and modulates its activity. J Biol Chem 279: 17329-17337, 2004.

22. Bourguignon LY, Gilad E, Rothman K and Peyrollier K: Hyaluronan-CD44 interaction with IQGAP1 promotes Cdc42 and ERK signaling, leading to actin binding, Elk-1/Estrogen Receptor transcriptional activation, and ovarian cancer progression. J Biol Chem 280: 11961-11972, 2005.

23. Briggs MW, Li Z and Sacks DB: IQGAP1-mediated stimulation of transcriptional co-activation by beta-catenin is modulated by calmodulin. J Biol Chem 277: 7453-7465, 2002

24. Wang Y, Wang A, Wang F, Wang M, Zhu M, Ma Y and Wu R: IQGAP1 activates Tcf signal independent of Rac1 and Cdc42 in injury and repair of bronchial epithelial cells. Exp Mol Pathol 85: 122-128, 2008

25. Schmidt VA, Chiariello CS, Capilla E, Miller F and Bahou WF: Development of hepatocellular carcinoma in Iqgap2-deficient mice is IQGAP1 dependent. Mol Cell Biol 28: 1489-1502, 2008.

26. Nabeshima K, Shimao $Y$, Inoue $T$ and Koono $M$ : Immunohistochemical analysis of IQGAP1 expression in human colorectal carcinomas: Its overexpression in carcinomas and association with invasion fronts. Cancer Lett 176: 101-109, 2002.

27. Hayashi H, Nabeshima K, Aoki M, Hamasaki M, Enatsu S, Yamauchi Y, Yamashita Y and Iwasaki H: Overexpression of IQGAP1 in advanced colorectal cancer correlates with poor prognosis-critical role in tumor invasion. Int J Cancer 126: 2563-2574, 2010

28. Skrzypczak M, Goryca K, Rubel T, Paziewska A, Mikula M, Jarosz D, Pachlewski J, Oledzki J and Ostrowski J: Modeling oncogenic signaling in colon tumors by multidirectional analyses of microarray data directed for maximization of analytical reliability. PLoS One 5: e13091, 2010.

29. Livak KJ and Schmittgen TD: Analysis of relative gene expression using real time PCR and the 2 (-Delta Delta C(T)) method. Methods 25: 402-408, 2001.

30. Abel AM, Schuldt KM, Rajasekaran K, Hwang D, Riese MJ, Rao S, Thakar MS and Malarkannan S: IQGAP1: Insights into the function of a molecular puppeteer. Mol Immunol 65: 336-349, 2015.

31. Dong P, Nabeshima K, Nishimura N, Kawakami T, Hachisuga T, Kawarabayashi $\mathrm{T}$ and Iwasaki $\mathrm{H}$ : Overexpression and diffuse expression pattern of IQGAP1 at invasion fronts are independent prognostic parameters in ovarian carcinomas. Cancer Lett 243: $120-127,2006$

32. Chen F, Zhu HH, Zhou LF, Wu SS, Wang J and Chen Z: IQGAP1 is overexpressed in hepatocellular carcinoma and promotes cell proliferation by Akt activation. Exp Mol Med 42: 477-483, 2010.

33. Wang XX, Li XZ, Zhai LQ, Liu ZR, Chen XJ and Pei Y: Overexpression of IQGAP1 in human pancreatic cancer. Hepatobiliary Pancreat Dis Int 12: 540-545, 2013.

34. Smith JM, Hedman AC and Sacks DB: IQGAPs choreograph cellular signaling from the membrane to the nucleus. Trends Cell Biol 25: 171-184, 2015

35. White CD, Brown MD and Sacks DB: IQGAPs in Cancer: A family of scaffold proteins underlying tumorigenesis. FEBS Lett 583: 1817-1824, 2009.

36. Jameson KL, Mazur PK, Zehnder AM, Zhang J, Zarnegar B, Sage J and Khavari PA: IQGAP1 scaffold-kinase interaction blockade selectively targets RAS-MAP kinase-driven tumors. Nat Med 19: 626-630, 2013.

37. Pi F, Binzel DW, Lee TJ, Li Z, Sun M, Rychahou P, Li H, Haque F, Wang S, Croce CM, et al: Nanoparticle orientation to control RNA loading and ligand display on extracellular vesicles for cancer regression. Nat Nanotechnol 13: 82-89, 2018. 
38. Zhao CY, Cheng R, Yang Z and Tian ZM: Nanotechnology for cancer therapy based on chemotherapy. Molecules 23: E826, 2018.

39. Awasthi R, Roseblade A, Hansbro PM, Rathbone MJ, Dua K and Bebawy M: Nanoparticles in cancer treatment: Opportunities and obstacles. Curr Drug Targets 2018.

40. Rychahou P, Bae Y, Reichel D, Zaytseva YY, Lee EY, Napier D, Weiss HL, Roller N, Frohman H, Le AT and Mark Evers B: Colorectal cancer lung metastasis treatment with polymer-drug nanoparticles. J Control Release 275: 85-91, 2018.
41. Marquez J, Fernandez-Piñeiro I, Araúzo-Bravo MJ, Poschmann G, Stühler K, Khatib AM, Sanchez A, Unda F, Ibarretxe G, Bernales I and Badiola I: Targeting liver sinusoidal endothelial cells with miR-20a-loaded nanoparticles reduces murine coloncancer metastasis to the liver. Int J Cancer 143: 709-719, 2018.

42. Li Y, Duo Y, Bi J, Zeng X, Mei L, Bao S, He L, Shan A, Zhang Y and $\mathrm{Yu}$ X: Targeted delivery of anti-miR-155 by functionalized mesoporous silica nanoparticles for colorectal cancer therapy. Int J Nanomedicine 13: 1241-1256, 2018. 